\title{
LPC for Signal Analysis in Cellular Network Coverage
}

\author{
Koffi Agbeblewu Dotche, François Sekyere, Walter Banuenumah \\ Department of Electrical \& Automotive Technology Education, Faculty of Technical Education, College of \\ Technology Education, University of Education Winneba (UEW), Kumasi, Ghana \\ Email: kdotche2004@gmail.com
}

Received 23 May 2016; accepted 17 July 2016; published 20 July 2016

Copyright (C) 2016 by authors and OALib.

This work is licensed under the Creative Commons Attribution International License (CC BY). http://creativecommons.org/licenses/by/4.0/

(c) $\underset{E Y}{0}$ Open Access

\section{Abstract}

This paper introduces a novelty method of using a Linear Prediction Coefficient (LPC) filter, a digital signal processing (DSP) tool to get accurate signal measurement in noisy mobile environment. By measuring the received power of a mobile radio, it also measures the coverage of an area served by several base stations. For results' validation, the mobile received power at user end of two Code Division Multiple Acccess-2000 (CDMA2000) cellular networks operating at different frequency (450 MHz and $800 \mathrm{MHz}$ ) in the same environment, Lome in Togo, was considered. Our analysis has consistently shown that within the problem areas in the coverage, the filter response does not match with the measured data. These mismatching areas may likely result from poor soft-handoff process or some dead zones. The study has proven the significant help of this novelty method in problem areas identification. Consequently, such a filter can be embedded to current firmware for Radio Frequency coverage optimization, and for an effective spectrum efficiency.

\section{Keywords}

Radio Channel Characteristics, Received Power, Coverage Analysis, Linear Coefficient Prediction (LPC), Drive Test

\section{Subject Areas: Electric Engineering}

\section{Introduction}

Radio coverage estimation is very costly for a mobile cellular operator. It may be admitted that it is the most perceived criterion of performance by mobile users. In order to add value and save their investment, the network performance may constantly be monitored. The cellular network coverage performance is thus impaired by the network design, the continuous optimization process and the surrounding clutter at both the Mobile Station (MS) and the Base Station (BS). The clutter constitutes the radio space channel. It is defined as the air interface be- 
tween the Mobile Station (MS) and the Base Station (BS or BTS). It takes into account buildings, trees, streets, hills, water; and all kinds of obstructions in addition to the extent of the terrain's roughness, etc. In this environment, the signal is heavily influenced by reflections from buildings. The clutter effect on the radio signal received at MS inflects the signal to vary randomly. This variation in received signal is quantified by the effect of shadowing or environment fading that is mainly depended on the mobile user location.

The fading envelope of a received signal strength may be viewed as having two main variant components [1], the fast fading signal observed over small distance (known as Small Scale Fading: SSF) and slow fading signal as over a long distance (known as Large Scale Fading: LSF or Slow Fading). The large-scale fading is related to diffraction and reflection effects that the multipath components undergo on their way between the transmitter and the receiver. The small-scale fading or fast fading is rapid changes observed in received signal. These variants were caused by the user mobility [2]. The fast fading magnitude was obtained on an average number of finite sample measurements but the slow fading has consisted to carry out an average of wide measurement over long distances [1]. On the other hand, another general phenomenon, which is a source of signal attenuation, is multipath propagation. Multipath propagation has been analyzed by several stochastic models [3] [4], available in literature. When all multipath signals reach the receiver with same levels); in this particular case, there is no direct rays that reach the receiver, the Rayleigh fading model is applicable. Another type of channel fading is the Rice fading model. In this type of channel fading, one multipath signal is stronger than the rest; a direct ray may likely reach the receiver point. Thus, it is appropriate for line-of sight channel. Both fading models have been generalized in the Nakagami fading model [5]. However, the area of radio channel estimation remains broad to investigate; this includes the modern cellular network coverage analysis.

Cellular network coverage analysis is a delicate task for the Radio Frequency (RF) planning engineer at deployment stage as well as the optimization phases. Several tools for RF coverage analysis in cellular networks are available on the market. These tools, such as Agilent analyzers, cell-test, master-site, Airbridge and so on; perform channel measurement adequately. But then, it may be admitted that these tools perform statistical analysis of the radio network coverage either per base station or per sector with regard to a predefined threshold. Recently, radio channel estimation and its components analysis have gained attention in using a digital signal processing (DSP) tool to get accurate channel estimation as in [6]. The work has proposed a dynamic adaptive estimator that comprises the spline and smoothing spline tools to discriminate path loss components on received signal measurement, such as path loss, shadowing and fast fading. This method debunks from the statistical methods used for path loss components estimation as in [7]-[9], a communication channel tracking apparatus has been proposed that made used a linear predictor a DSP tool for partial channel estimation with finite time sample.

The study in this paper introduces a novelty method for cellular coverage analysis by using a Linear Prediction Coefficient (LPC) filter [10], a DSP tool to investigate the mobile handset received power level in noisy environment. The received power measurements have been performed on field installation of two different Code Division Multiple Access-2000 (CDMA-2000) cellular networks operating in the same environment in Lome, Togo. One is operating at $450 \mathrm{MHz}$ and the second network is operating at $800 \mathrm{MHz}$. Our contribution remains in the light of exploring dynamic filter for radio channel estimation.

The rest of the paper is structured as follows: Section 2 deals with the linear predictor as coverage estimator and the theory behind, the data collection using the drive testing is presented in Section 3; the comparative results between the estimator response and the measured data are developed in Section 4; and Section 5 concludes and leads to future works.

\section{Linear Predictor (LPC) as Coverage Estimator}

In this section the theory of the shadowing with frequency dependency and the lpc as the coverage estimator in noisy mobile environment are addressed.

\subsection{Received Power and Shadowing Frequency Dependency}

The received power $p_{R_{X}}$ at MS as function of the frequency [4] is as given in (1):

$$
p_{R_{X}}=p_{T_{X}} * g_{T_{X}} * g_{R_{X}} *\left(\frac{c}{4 * \pi}\right)^{n} *\left(\frac{1}{d}\right)^{n} *\left(\frac{1}{f}\right)^{n} * x_{\sigma} .
$$


Let $l$ (the path loss) define the ratio of transmitted power $p_{T_{X}}$ to the received power $p_{R_{X}}$ in watt scale. Though, by convention the path loss $L(\mathrm{~dB})$ is always expressed in $\mathrm{dB}$ scaleas the observed difference in the base station transmitted power $P_{T_{x}}[\mathrm{~dB} \cdot \mathrm{m}]$ and the received power $P_{R_{x}}[\mathrm{~dB} \cdot \mathrm{m}]$ at mobile handset in mobile communication environment [3] [4]. Thus, we can write:

$$
\left\{\begin{array}{l}
l=-\frac{p_{R_{x}}}{p_{T_{x}}} \Rightarrow \\
L(\mathrm{~dB})=-10 * \log _{10}\left(\frac{k}{f^{n}} * x_{\sigma}\right)
\end{array}\right.
$$

where

$$
\begin{gathered}
k=g_{T_{X}} * g_{R_{X}}\left(\frac{c}{4 * \pi}\right)^{n} *\left(\frac{1}{d}\right)^{n} . \\
K(\mathrm{~dB})=G_{T_{X}}+G_{R_{X}}+10 n \log _{10}\left(\frac{c}{4 \pi}\right)-10 n \log _{10} d \\
L(\mathrm{~dB})=K-10 n \log _{10}(f)+X_{\sigma}(\mathrm{dB}) .
\end{gathered}
$$

$p_{T_{X}}$ is the transmitting antenna power and transmitting antenna gain $g_{T_{X}}$,

$g_{R_{X}}$ is the user handset (MS) antenna gain,

$n$ the path loss exponent that describes the environment,

$d$ distance of separation between a BS and an MS

$x_{\sigma}$ is the shadowing effect of the environment,

$K$ is a measure of the local mean variation in the large scale sense and its distance dependency,

$\lambda=c / f$ is the wavelength, $f$ is the carrier frequency, and $c$ is the speed of light in vacuum,

$L$ is the path loss having three dependency parameters $f, d$ and $n$.

If we assume that shadowing can be viewed as a correlated error on the measurement in a time varying process and can be derived as a frequency dependency function as shown in (6):

$$
X_{\sigma}=L(\mathrm{~dB})-K(\mathrm{~dB})+10 n \cdot \log _{10} f .
$$

It comes as in (7)

$$
\left\{\begin{array}{l}
X_{\sigma} \propto C+10 n \cdot \log _{10} f \\
C, \text { cst }
\end{array}\right.
$$

$X_{\sigma}$ is therefore frequency and environment dependency.

\subsection{Linear Predictive Coefficient (LPC)}

The LPC tool determines the coefficients of a forward linear predictor by minimizing the prediction error in the least squares sense (Figure 1). It has applications in filter design, speech coding, and system identification [11]. In system analysis, linear predictive coding is known as a mathematical optimization process. In Matlab software [12], the function $\operatorname{lpc}(x, p)$ finds the coefficients of a $p$ th-order linear predictor (FIR filter) that predicts the current value of the real-valued time series $x$ based on past samples. Where, the predicted signal $\hat{x}(n)$ is given as in (8):

$$
\hat{x}(n)=\sum_{i=1}^{p} a_{i} x(n-i)
$$

where $p$ is the order of the prediction filter polynomial, and $a_{i}=[1, a(2), \cdots, a(p+1)]$, is the predictor coefficient.

with the predictor error $e(n)$ given as in (9) and (10)

$$
e(n)=x(n)-\hat{x}(n)=x(n)-\sum_{i=1}^{p} a_{i} x(n-i)
$$




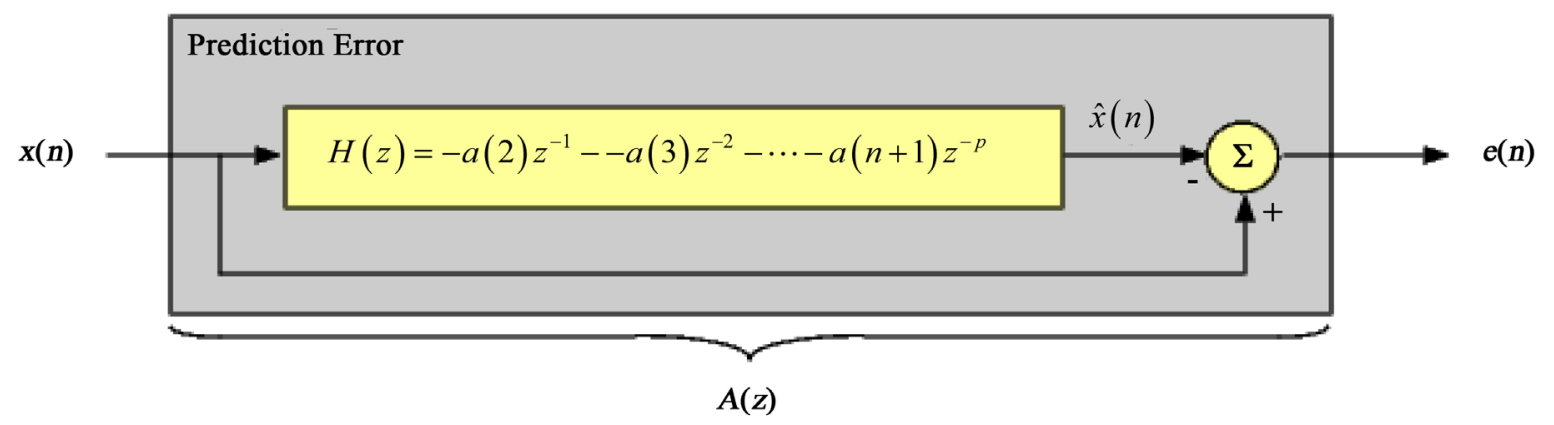

Figure 1. The LPC predictor block [12].

$$
e(n)=-\sum_{i=0}^{p} a_{i} x(n-i)
$$

It may be noted that $a_{i}$ is the autocorrelation criterion. For $a_{i}=1$ the predictor is linear filter.

The prediction error $e(n)$ can be viewed as the output of the prediction error filter $A(z)$ as shown in Figure 1 , where $H(z)$ is the optimal linear predictor, $x(n)$ is the input signal.

\subsection{Signal Components and Coverage Estimation}

In Figure 2, it is showing the linear adaptive filter incorporated with the LPC tool, to perform the radio signal measurement in cellular network coverage in multi-transmitter environment. For a given data, one may estimate the path loss components, and evaluate the coverage analysis as whole through the LPC filter.

The fast fading estimation is achieved by computing the Robust Lowess (linear fit) algorithm. This is an advanced smoothing spline method. Meanwhile, the slow fading is obtained by using the Robust Loess (Quadratic fit) algorithm [13]. However, the local mean is obtained by subtracting the fast fading quantity out of the slow fading value.

\subsection{Channel Estimation}

The RMS value of the $j$ th column of an M-by-N input matrix $u$ is given by

$$
y_{j}=\sqrt{\frac{\sum_{i=1}^{M}\left|u_{i j}\right|^{2}}{M}}
$$

where $1 \leq j \leq N$.

The root mean square error (RMSE).

This statistical parameter measures the total deviation of the response values from the fit to the response values. It is also called the summed square of residuals and is usually written as SSE.

The fade margin is computed by the formula given as:

$$
M=\sqrt{\frac{\sum_{i=1}^{M}\left|R_{i j}-R_{t h r}\right|^{2}}{M}}
$$

$R_{i j}$ the signal at a point $(i, j)$,

$R_{t h r}$ the signal threshold.

\section{Methodology of Data Collection}

The measurement experiment consisted of performing a simultaneous drive testing in two cellular networks operating at two different frequencies with two CDMA Handsets (Huawei C5320 for $450 \mathrm{MHz}$ and Kyocera Kx5 for $850 \mathrm{MHz}$ ). The $450 \mathrm{MHz}$ network has known a recent extension. The second network of $850 \mathrm{MHz}$ is not loaded during the test and recently deployed.

Both networks are operating in Lome and belong to the same operator (Togo Telecom). 


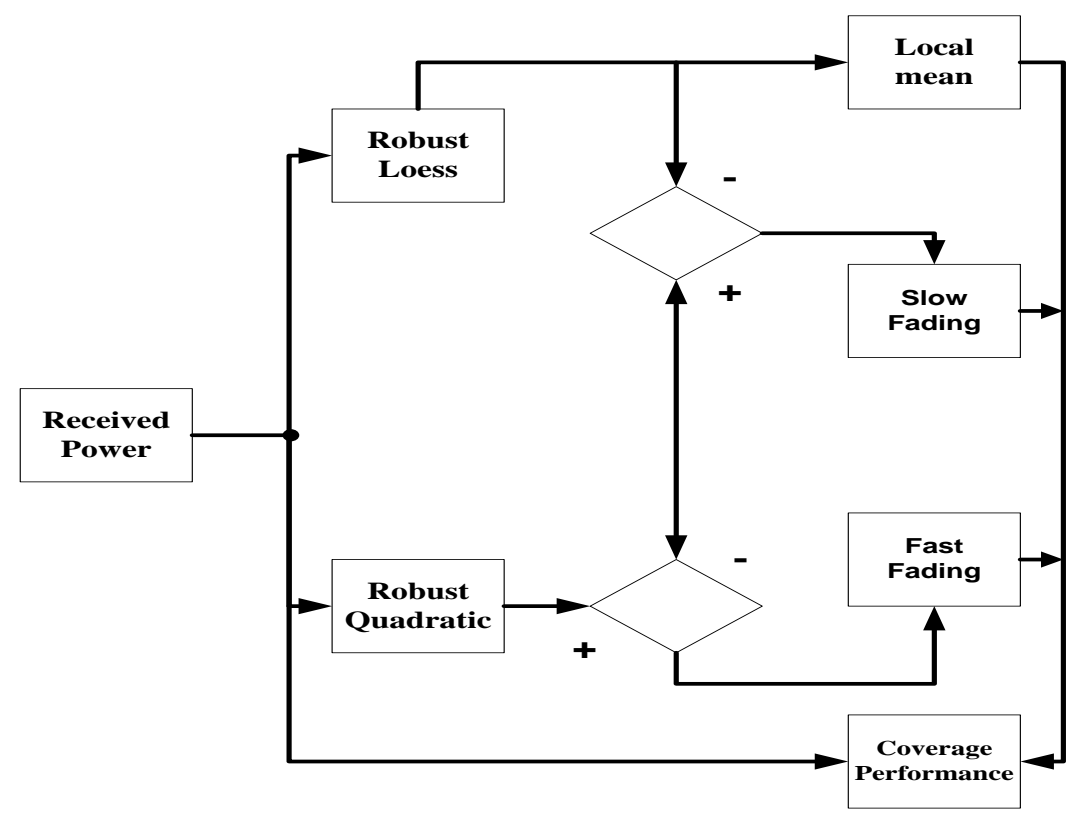

Figure 2. Linear adaptive filter flow chart.

The setup of the measurement procedure is illustrated in Figure 3 . The drive testing is carried out while roaming in the wireless cellular network with over-air test (OAT) tools by an equipped car. These tools are: a Global Positioning System (GPS) receiver antenna placed in the car rooftop (to obtain the accurate distance of location) and GPS 76 software version 2; a drive-test software (ZK CellTest tool [14] capable to to read the dongle (see Figure 3) and a communication network analyser software (here, the Gladiator firmware for extracting the call log traces after the drive-testing is completed, assigned in the office), a laptop (the client), Map info software (for loading the digital map); Inverter (for converting the DC source obtained from the car inner-port to the AC source with purpose of supplying power to the laptop in case of a long time drive testing); an Extension board (for connecting the laptop, and various hardware that need AC current).

The inverter in Figure 3 is meant for converting the Direct Current (DC) voltage at the output of the connector to Alternative Current (AC) (220). This is used to supply sufficient power to the laptop during a whole day drive test. For very a few minutes' drive-testing, the inverter may not be necessary. The Mobile Equipment line (M1) will be calling the Mobile Equipment line (M2). In some cases, the line (M2) can be assigned to a fixed line office. In this case, one mobile may be used for the drive test.

The drive test traces' map is showing in Figure 4 as in multi-transmitters' system in a noisy mobile cellular environment.

The measurement was a simultaneous and continuous in both operating frequencies of the transmitters, in 450 $\mathrm{MHz}$ and $850 \mathrm{MHz}$. The extracted data from the Gladiator firmware was processed to Matlab software and been analyzed by the usage of the lpc estimator.

\section{Results and Discussion}

For a fixed transmitter, the robust linear fit method is used with the help of the "cftool" in Matlab software for the estimation of the path loss components as shown in Figure 5.

In Figure 5, it is showing the path loss components obtained using the robust loess linear fit to quantify the slow fading on the measurement. The robust quadratic fitting shows the fast fading components on the measurement. The obtained plots show a better agreement with the measurement data as compared to the simple smooth spline method proposed in [6].

In Figure 6, a sample for the received signals during the measurement in both frequencies is showing the attenuation effect in the signal due to the frequency dependency.

Figure 7 is depicting the received signal level being processed as a time series to the adaptive filter proposed 


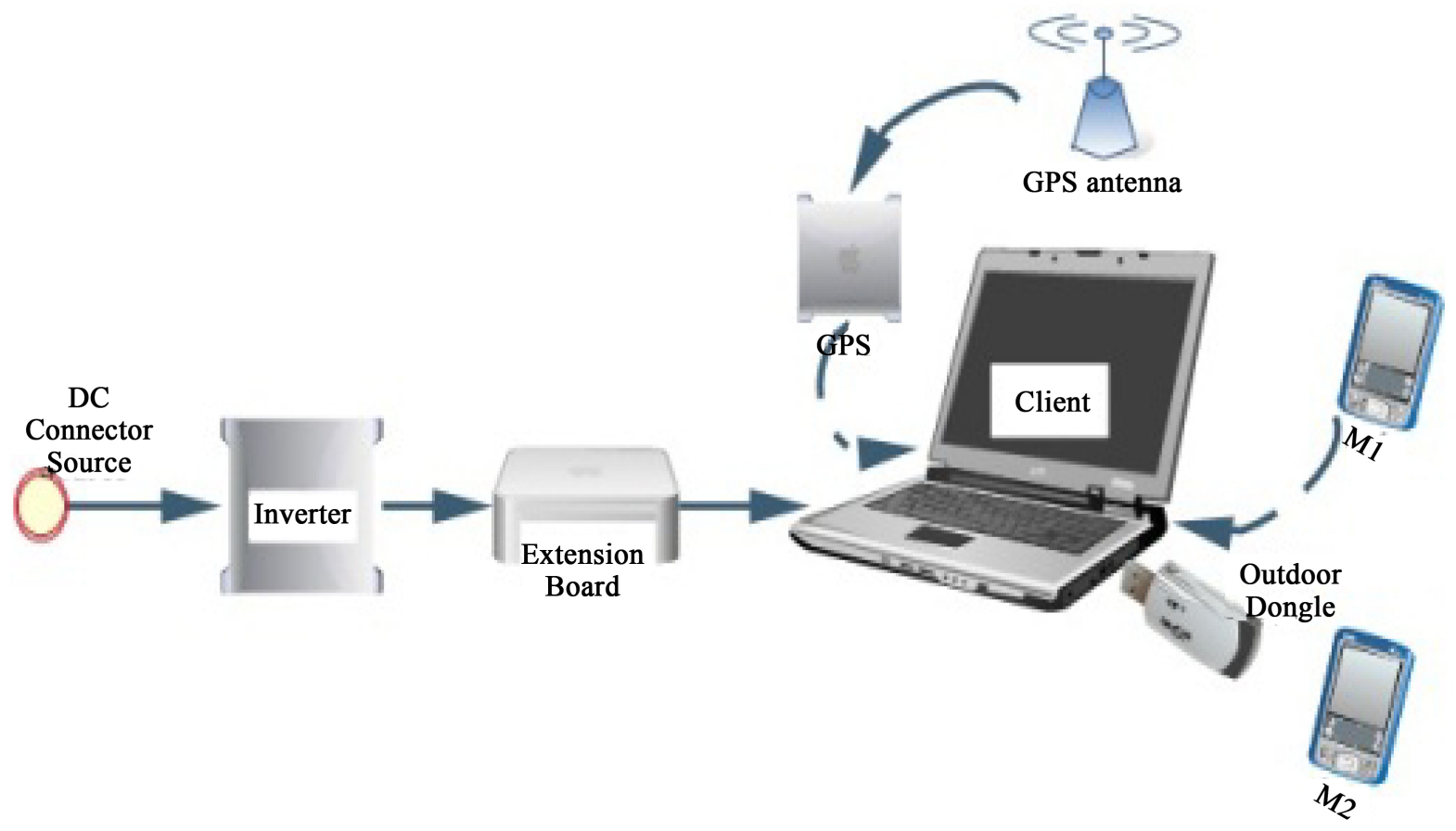

Figure 3. Drive test setup system.

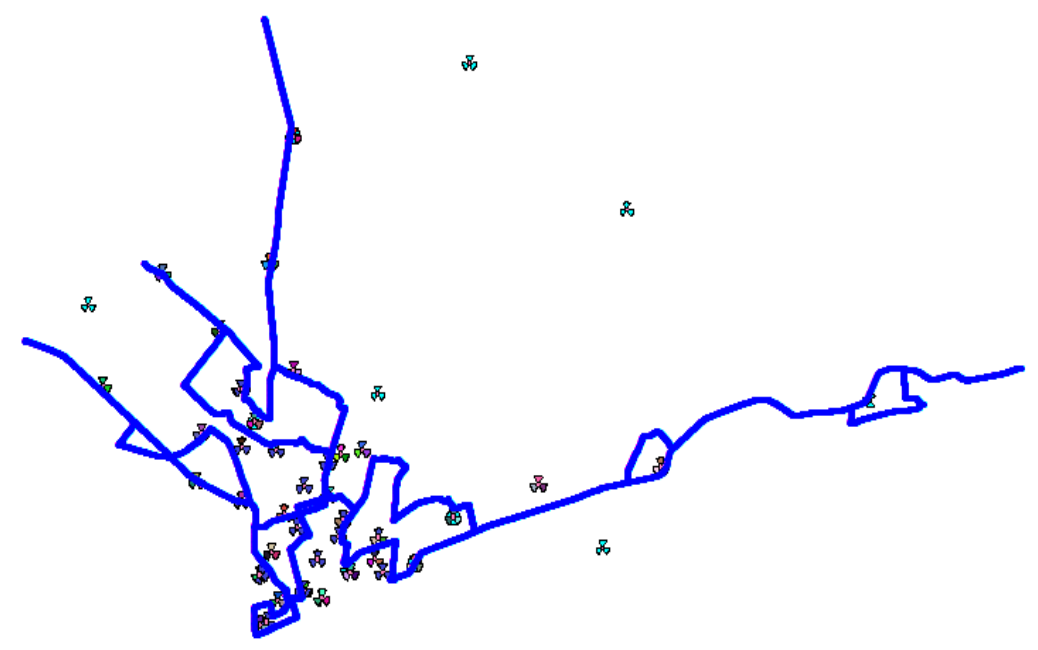

Figure 4. Drive test road map.

in Figure 2 for the coverage performance estimation.

In Figure 7, the measured data compared to the filter response in both frequencies in $450 \mathrm{MHz}$ and $850 \mathrm{MHz}$.

In $850 \mathrm{MHz}$ the Linear PLC filter matches with the measurement as shown in Figure 7. This may be explained by the fact that the transmitters in used are sufficiently enough for the intended coverage. Aside this, the network load may be termed as low. Therefore the system margin gain is seen to be reliable for the soft-handoff. The channel impairments such as the shadowing and multipath components are markedly compensated (reduced) because of the use of directional antennae and the down-tilt technique [15].

In $450 \mathrm{MHz}$, the network may present some deficiency such as persistent dead zone thus dropped calls as illustrated in Figure 7. This may show an inconstancy of dimensioning at design process. Consequently it may be explained by the fact that on one hand, the distance of separation among Base Transceiver Sub-system often called Base Station (BTS/BS) is not well set thus the cell overlapping is large at some areas. Meanwhile on the other hand, this may also be viewed as due to the extension of the network. Particularly, adding new Pseudo 


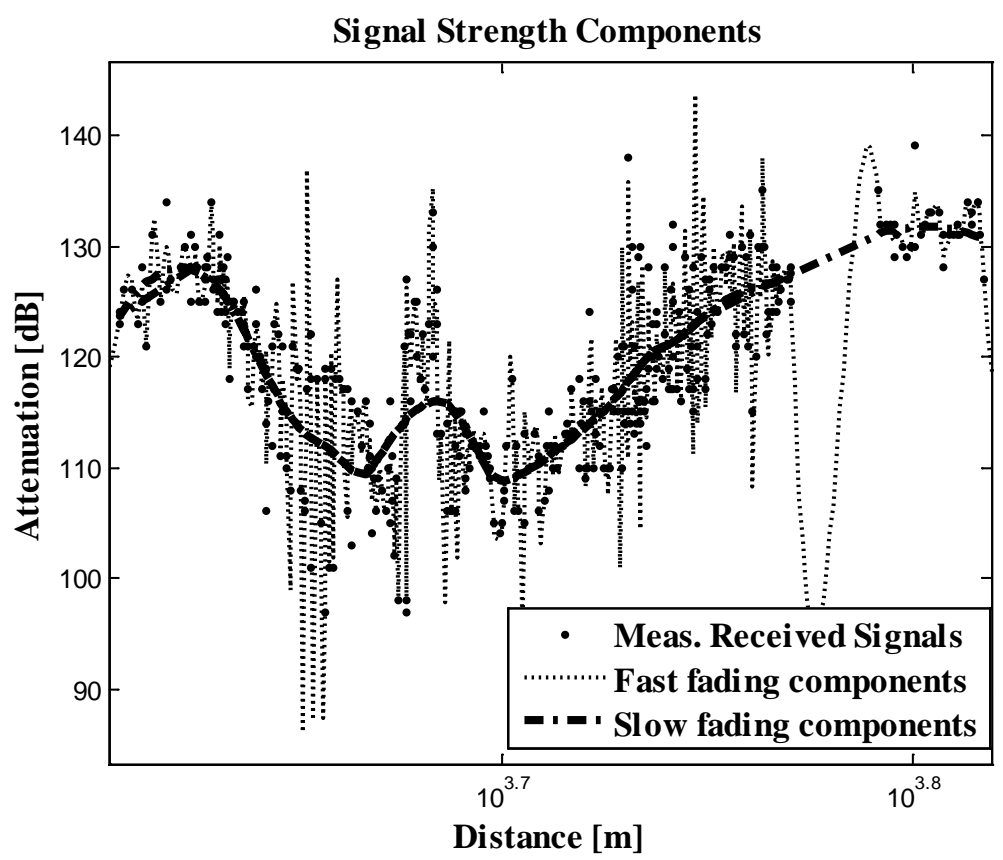

Figure 5. Received signal components in mobile cellular environment.

Path Loss Effect and Frequency in Lome

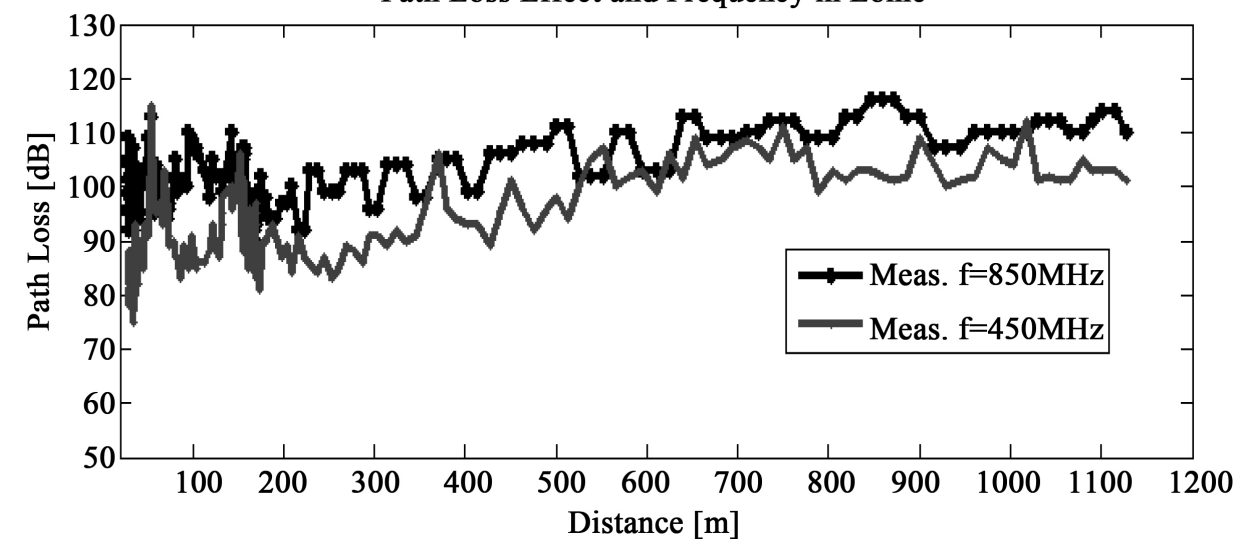

Figure 6. Comparison of received signals.

Noise (PN) code [16] is also a delicate process to an existing network.

A poor evaluation of PN may highly create interference in the network coverage area. These interference areas observed may result in unnecessary soft-handoff at times.

This may also be explained by the size of the soft-handoff window-search [17]. In this particular case, if the size of the soft-handoff window-search is small by then unnecessary soft-hand may also occur even at a relative short distance of the potential BTS. It may therefore be argued that the small mismatching areas are some areas of poor soft-handoff, whereas the large mismatching areas may be resolved in an extremely poor hard handoff decision due to high interference from other non-CDMA antennae. Though the antennae' heights of $450 \mathrm{MHz}$ BTSs are mostly the highest as compared to others sharing the same mast thus users at cell-edge may observe high interference. This situation may then lead to call drops in this mismatchingas depicted by the filter response. The advantage observed in the filter response is that, these areas can be easily localized by tracing back the log files.

Theoretically, it may also be presumed that the response of filter may show a little agreement in $850 \mathrm{MHz}$, which could reveal the effect of shadowing effect in high frequency. In fact the low network load status may 

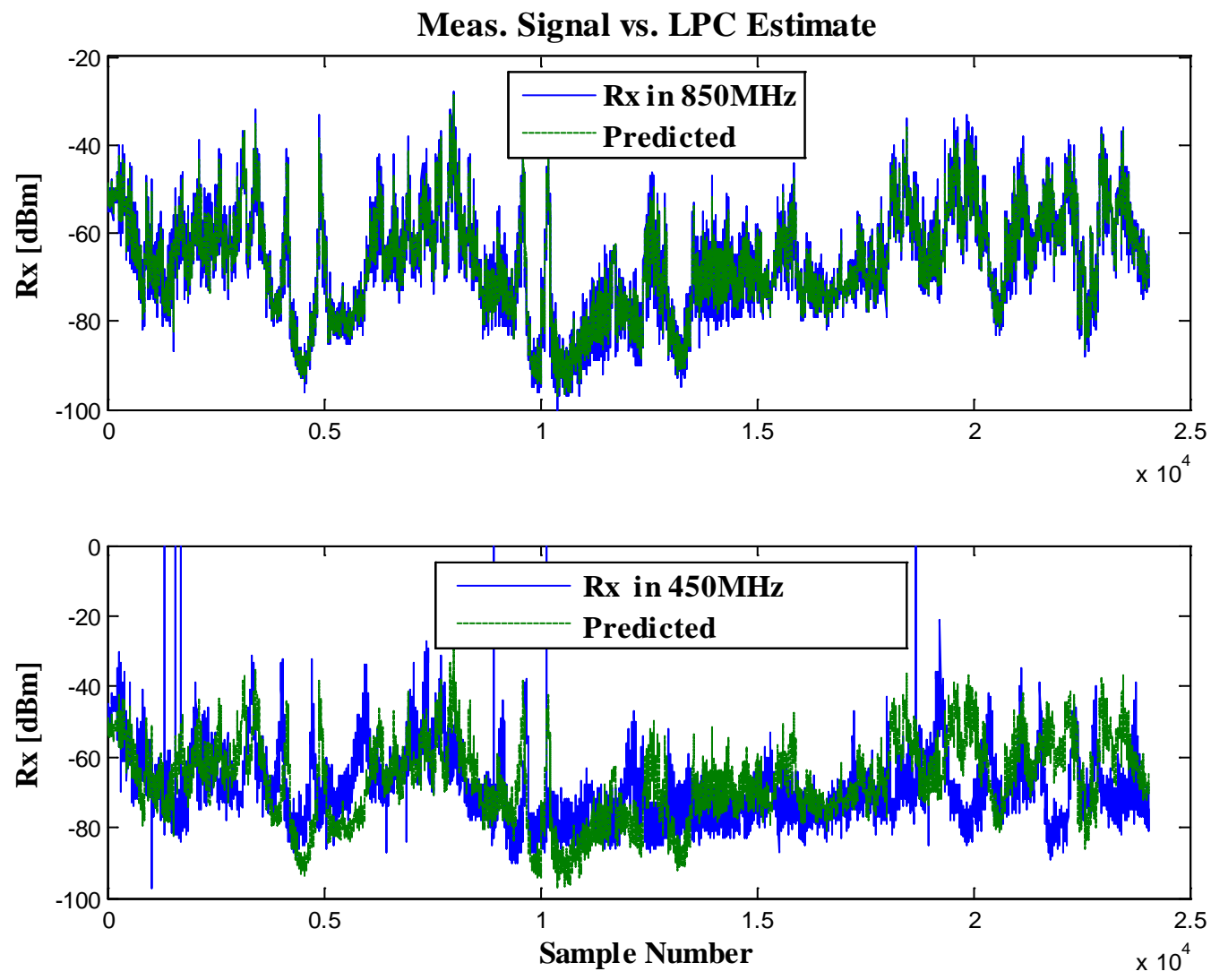

Figure 7. Measured Signals compared with LPC signals in $850 \mathrm{MHz}$ and $450 \mathrm{MHz}$ respectively.

rather prevail. It may be pointed out in turns that, the LPC has adjusted the past measurements to the forward measurement that may have shown agreement with the real measurement. Furthermore, the analysis of the autocorrelation of the predictor error in Figure 8, may reveal that the shadowing may be viewed as an error on the received signal and thereby may be approximated to a white Gaussian noise. Therefore, a shadowing effect in cellular environment may also be modeled as a white Gaussian noise.

Subsequently, the computation of the standard deviation of both data (the raw data and the data at the output of the LPC), the shadowing attenuation is obtained. By carrying out the root mean square error on both data, this gives us the fast fluctuation component. The results are presented in Table 1.

The results in Table 1 may indicate that the effect of shadowing and the path loss exponent may seem higher in $850 \mathrm{MHz}$ than that of $450 \mathrm{MHz}$. Subsequently this may be explained by the lower shadowing effect at a lower frequency. In the study environment of Lome, its attenuation value was found to be $9 \mathrm{~dB}$ in $450 \mathrm{MHz}$ and 11.5 $\mathrm{dB}$ in $850 \mathrm{MHz}$. It may also be perceived that the local meanvariation was found not to be a frequency dependency. With respect to the deviation or the difference in value observed of the measured data and that of the LPC processed data, this value is constant with an error margin less than $0.5 \mathrm{~dB}$.

\section{Conclusions}

In this paper, we have proposed a novel feasibility of RF network coverage performance analysis using a digital signal processing tool, the LPC filter. The LPC filter is often used in speech coding. This filter was applied to analyze the received power obtained from two CDMA2000 cellular networks that are operating at different frequencies but servicing in the same environment. The analysis has shown that within the problem areas the filter response does not match with the measured data. These mismatching areas may be due to either some poor soft handoffs, or unnecessary soft handoffs, or even some dead zone spots in the coverage area.

Such a tool can be embedded to some RF coverage analysis network tools, as a case for cognitive radio. This may greatly help in problem areas identification, such as dead zone and poor soft-handover in cellular network 

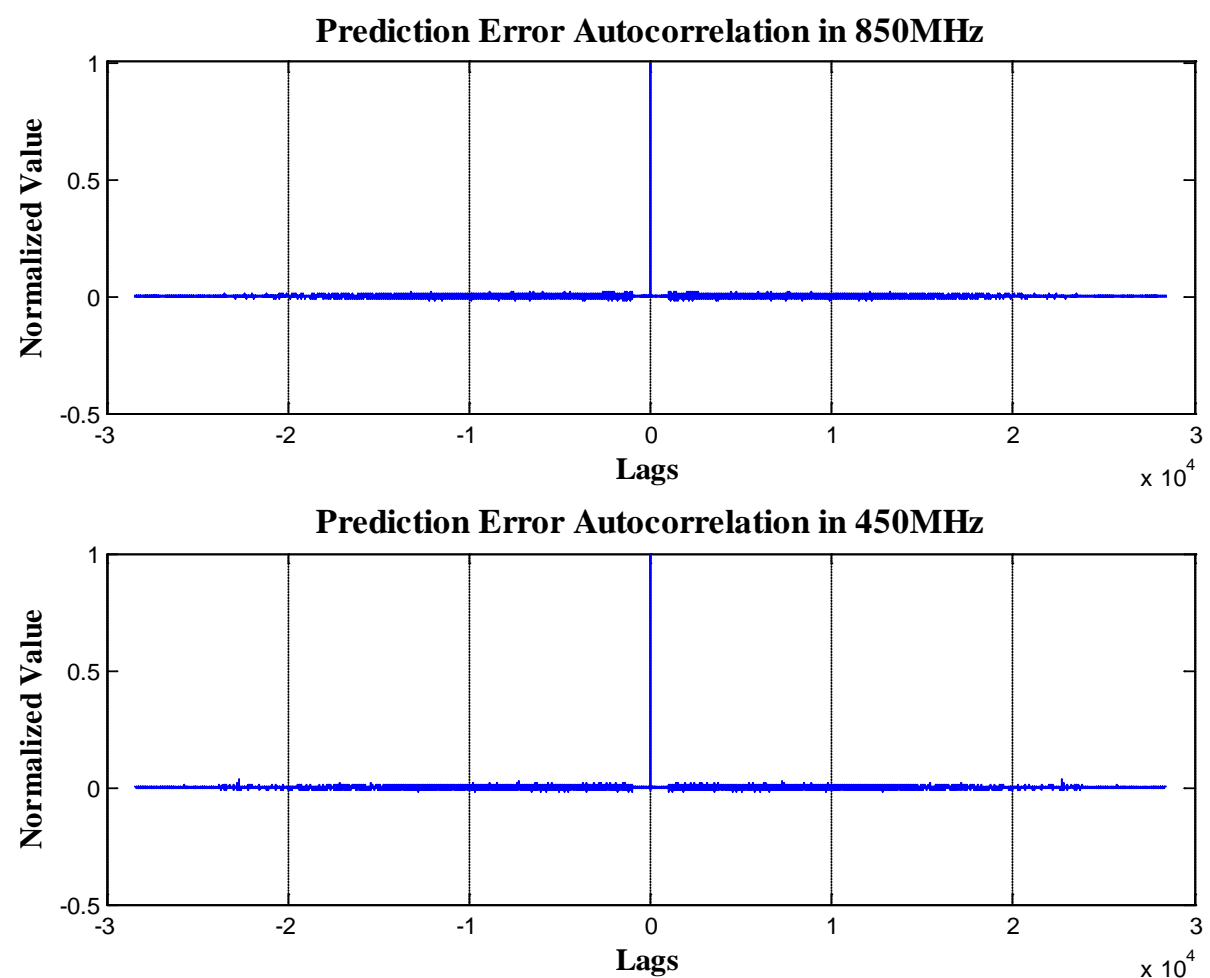

Figure 8. View of problem areas and LPC predicted data.

Table 1. Radio channel characteristics.

\begin{tabular}{|c|c|c|c|c|}
\hline & \multicolumn{2}{|c|}{$850 \mathrm{MHz}$} & \multicolumn{2}{|c|}{$450 \mathrm{MHz}$} \\
\hline & Raw & LPC & Raw & LPC \\
\hline Location mean variation in the near field $(\mathrm{dB}) \mathrm{K}$ & 7.32 & 8.213 & 6.93 & 8.1367 \\
\hline Effect of shadowing (dB) $\mathrm{X}$ & 11.6989 & 11.3330 & 10.1630 & 9.6567 \\
\hline Fast fluctuation $n$ & 3.22 & 3.12 & 2.19 & 1.52 \\
\hline
\end{tabular}

coverage.

\section{Acknowledgements}

We wish to express our gratitude, to the anonymous reviewers for their valuable contributions, and Olivier Gibaudau at Global-Tech France, for their technical support during the measurement campaign.

\section{References}

[1] Lee, W.C.Y. (1985) Estimate of Local Average Power of a Mobile Radio Signal. IEEE Transactions on Vehicular Technology, 34, 22-27.

[2] Egli, J. (1957) Radio Propagation above 40 MC Over Irregular Terrain. IEEE Transaction on Vehicular Technology, New York.

[3] Parsons, J.D. (1992) The Mobile Radio Propagation Channel. 2nd Edition, John Wiley and Sons, London.

[4] Rahnema, M. (2008) UMTS Network Planning, Optimization and Inter-Operation with GSM. John Wiley \& Sons Asia Pte Ltd.

[5] Oestges, et al. (2009) Experimental Characterization and Modeling of Outdoor-to-Indoor and Indoor-to-Indoor Distributed Channels. Technical Report, European Cooperation in the Field of Scientific and Technical Research, Braunschweig. 
[6] Le Cornec, et al. (2007) Optimization of Radio Measurements Exploitation in Wireless Mobile Networks. Journal of Communications-JCM, 2, 59-67.

[7] Adebayor, et al. (2006) Characterization of Propagation Path Loss at 1.8 GHz: A Case Study of Benin-City, Nigeria. Journal of Applied Sciences, Medwell Online, 1, 92-96.

[8] Konak, A. (2010) Estimating Path Loss in Wireless Local Area Network Using Ordinary Kriging. Proceedings of the 2010 Winter Simulation Conference, Pennsylvania. http://www.informs-sim.org/wsc10papers/267.pdf

[9] Gifford, et al. (2008) A Communication Channel Tracking Apparatus. US7433434 B2.

[10] Wikipedia Encyclopedia. http://en.wikipedia.org/wiki/Linear prediction

[11] Jackson, L.B. (1989) Digital Filters and Signal Processing. 2nd Edition, Kluwer Academic Publishers, 255-257. http://dx.doi.org/10.1007/978-1-4615-3262-0

[12] MathWorks. Matlab Software Version 8. http://www.mathworks.com/help/techdoc/rn/f14-998197.html

[13] Ronaldo, D. Nonparametric Regression: Lowess/Loess. http://www.ime.unicamp.br/ dias/loess.pdf

[14] ZK Celltest (2009) http://zkcelltest.com/support/.../manual 10.4-1.pdf

[15] Dotche, K.A. and Diawuo, K. (2011) Received Power in Antenna Downtilt in Cluttered Mobile Environments: A Case Study of West-Africa. VDM, London.

[16] Ganesh, R. (2007) Impact of Adding Sites on PN Offset Planning in CDMA Networks. GTE Laboratories Incorporated, USA.

[17] Yang, C.S. (1998) CDMA RF Engineering. Artech House, Inc.

- Publication on a daily basis

Open Access Library

- 9 subject areas of science, technology and medicine

- Fair and rigorous peer-review system

- Fast publication process

- Article promotion in various social networking sites (LinkedIn, Facebook, Twitter, etc.)

- Widely-targeted and multidisciplinary audience to read your research

Submit Your Paper Online: Click Here to Submit

Contact Us: service@oalib.com 